\title{
Kinetics of immunoassays with particles as labels: effect of antibody coupling using dendrimers as linkers
}

\author{
Vladimir Gubala*a $^{* a}$, Carol Crean ${ }^{\mathrm{a}}$ (née Lynam), Robert Nooney ${ }^{\mathrm{a}}$, Stephen Hearty ${ }^{\mathrm{a}, \mathrm{b}}$, Barry McDonnell ${ }^{\mathrm{a}, \mathrm{b}}$, \\ Katherine Heydon ${ }^{\text {a }}$, Richard O'Kennedy ${ }^{\text {a,b }}$, Brian MacCraith ${ }^{\text {a }}$, David E. Williams ${ }^{\text {a,c }}$ \\ ${ }_{5}$ Received (in $\left.X X X, X X X\right)$ Xth $X X X X X X X X X 20 X X$, Accepted $X t h X X X X X X X X X 20 X X$ \\ DOI: $10.1039 / b 000000 x$
}

In this communication, we report on poly(amido amine) dendrimers (PAMAM) as coupling agents for recombinant single-chain ( $\mathrm{ScFv}$ ) antibodies to nanoparticle (NP) labels, for use in immunoassay. We present a simple theory for the kinetics of particle capture onto a surface by means of an antibody-antigen 10 reaction, in which the important parameter is the fraction of the particle surface that is active for reaction. We describe how increasing the generation number of the linking dendrimer significantly increased the fraction of the NP surface that is active for antigen binding and consequently also increased the assay kinetic rates. Use of dendrimers for conjugation of the NP to the antibody resulted in a significantly higher surface coverage of active antibody, in comparison with mono-valent linker chemistry. As a direct 15 consequence, the increase in effective avidity significantly out-weighed any effect of a decreased diffusion coefficient due to the NP, when compared to that of a molecular dye-labelled antibody. The signal to noise ratio of the G4.5 dendrimer-sensitised nanoparticles out-performed the dye-labelled antibody by approximately four-fold. Particle aggregation experiments with the multi-valent antigen CRP demonstrated reaction-limited aggregation whose rate increased significantly with increasing generation 20 number of the dendrimer linker.

\section{Introduction}

The context of this work involves the development of cardiac biomarker 'point of care' testing, which is predominantly based upon rapid immunoassay methods. ${ }^{1}$ The need for such 25 development is emphasised by the fact that almost half of the events associated with cardiovascular disease (CVD) occur without symptoms, ${ }^{2}$ while CVD is the cause of nearly half of all deaths in the western world. It is essential to maximise the signal to noise ratio to achieve clinically relevant sensitivity and limits 30 of detection in biomedical diagnostic devices ${ }^{3}$. Simple assay designs involve first capturing an antigen onto a surface using one antibody, then measuring the surface concentration by visualising the captured antigen through its reaction with a second, labelled antibody. Owing to their intense signal, 35 fluorescent nanoparticles (NP) are useful as labels since they can be measured directly, without the need for any amplification step 4-11. NP labels can, however, suffer from disadvantages in comparison with simple molecular labels, most notably effects due to particle aggregation ${ }^{12}$ and the related effect of non-specific ${ }_{40}$ binding ${ }^{13}$ to the capture surface. The benefits of using NPs can only be realized if they are efficiently coated with detection antibody, have good colloidal stability and the ratio of specific to non-specific binding (NSB) is sufficiently great. Another issue in the use of NPs as labels is the fraction of the coupled antibody
${ }_{45}$ that is in fact active or available for reaction with antigen ${ }^{14,15}$. This fraction can be rather small, which in turn can lead to diminished sensitivity and increased non-specific binding. The minimisation of NSB is essential for sensitive detection in an assay ${ }^{16}$. Thus, it is clear that the strategy used to attach an ${ }_{50}$ antibody to NP surface is a key element that affects the activity of the bound antibody, the non-specific binding and the surface binding of particles.

Improved colloidal stability of silica NPs has been previously achieved by the addition of negatively charged non-reactive 55 organosilanes in addition to organosilanes with functional groups available for bio-immobilisation ${ }^{12}$. The number of attachment sites on the nanoparticle surface is limited in order to maintain the suspension stability of the NP system. For this reason it is difficult to achieve high protein coupling ratios. We have recently ${ }_{60}$ demonstrated some advantages of using multivalent molecules such as dendrimers as antibody coupling scaffolds as illustrated on fig. $1 .{ }^{17}$ We have reported that good control over the colloidal stability of the NPs in the conjugation process can be achieved by using dendrimers as linkers. The use of dendrimers with $\mathrm{COOH}$ ${ }_{65}$ terminal groups had a positive effect on the monodispersity of the reaction mixture by maintaining a negative zeta potential of the individual particles. As a result, we were able to improve assay sensitivity and limits of detection, when compared to other NP samples prepared by using common homo- or hetero-functional 70 bilinkers. 
One of the specific reasons for exploiting antibody-sensitized dye doped nanoparticles as the next generation labels is their use in inexpensive and rapid, point-of-care biomedical diagnostics devices. In this context, the obvious questions we needed to 5 address were: i) what is the effect of the low diffusion coefficient of such large, dense fluorescent labels on the assay kinetics and how does the binding rate constant compare with the dye-labelled antibody?; and ii) what is the correlation between the active fraction area of NPs, varied by the use of different generation of 10 dendrimer, and the effect of NSB to the capture surface?

In this communication we present a simple kinetic theory for particle capture onto an antigen-loaded surface and compare the effect of using 4 sequential dendrimer generations to conjugate proteins to silica NPs containing a NIR fluorescent dye in their 15 core. We used as our model an assay for the stable biomarker Creactive protein, $\mathrm{CRP}$, whose serum concentration can increase 10,000-fold during cardiovascular inflammation. ${ }^{18} \mathrm{We}$ demonstrate significant improvements in the antigen-recognition activity of dendrimer-sensitised NPs, which correlates directly

20 with the generation number used for coupling and is consistent with the theory. We also show that the use of dendrimers as the coupling agents reduces NSB effects to less than the level exhibited by a molecular fluorophore-labelled antibody. The overall effect is a significantly improved signal/background ratio.

A
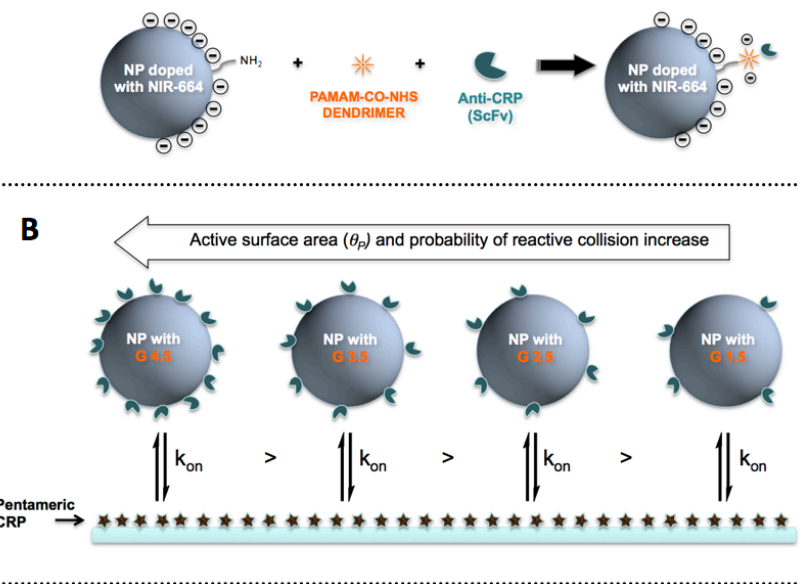

C
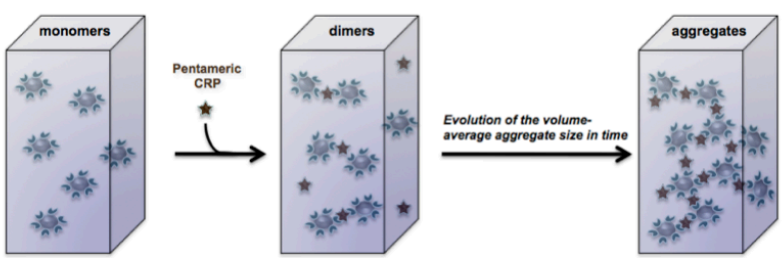

Fig. 1 (A) A cartoon showing the bioconjugation reaction between the nanoparticles and the recombinant single chain fragments $(\mathrm{ScFv})$ of antiCRP mediated by PAMAM dendrimers, (B) an illustration of a direct binding assay we used to demonstrate that increasing the active surface

30 area of the NP leads into increase in assay kinetic rates and (C) a graphic representation of the sensitized nanoparticle aggregation assay, induced by the introduction of the CRP antigen.

\section{Materials and Methods}

${ }_{35}$ Triton ${ }^{\circledR}$ X-100 (trademark Union Carbide), n-hexanol (anhydrous, $>99 \%$ ), cyclohexane (anhydrous $99.5 \%$ ), ammonium hydroxide ( $28 \%$ in $\mathrm{H}_{2} \mathrm{O}>99.99 \%$ ), tetraethylorthosilica (TEOS, $99.99 \%$ ), aminopropyltriethoxysilane (APTES, $99 \%$ \%), 3(trihydroxysilyl)propyl methyl phosphonate, monosodium salt 40 solution (THPMP, $42 \mathrm{wt} \%$ in water), absolute ethanol, monobasic sodium phosphate, dibasic sodium phosphate. phosphate buffered saline (PBS, pH 7.4, 0.01 M), Tween ${ }^{\circledR} 20$ (trademark Uniqema), sodium azide (99.99 \%), PAMAM dendrimers (-COONa surface groups), generations 1.5, 2.5, 3.5 45 and 4.5 (with diameters ranging from 1.5-4 nm) and albumin from bovine serum (BSA, $98 \%$ ) were all purchased from Sigma Aldrich Ireland, and used without further purification. Black 96 well plates used in the immunoassay were purchased from AGB Scientific Ireland. Deionised water $(>18 \mathrm{M} \Omega$ ) was obtained from 50 a Milli-Q system from Millipore Ireland. The microwell plates with array of $5 \times 15$ wells $\left(\mathrm{V}_{\text {well }}=10 \mu \mathrm{L}\right)$ were custom made by Micronit Microfluidics (Enschede, The Netherlands).

The dye used in this work is 4,5-Benzo-1'-ethyl-3,3,3',3'tetramethyl-1-(4-sulfobutyl) indodicarbocyanin-5'-acetic acid N55 succinimidyl ester, or more commonly referred to as NIR-664-Nsuccinimidyl ester (purchased from Sigma Aldrich). This dye has a quantum efficiency of $23 \%$, a molar absorptivity of $187,000 \mathrm{~L}$ $\mathrm{mol}^{-1} \mathrm{~cm}^{-1}$ and fluorescence excitation and emission wavelengths of $672 \mathrm{~nm}$ and $694 \mathrm{~nm}$, respectively, in isopropanol. ${ }_{60}$ Monodisperse nanoparticles $80 \mathrm{~nm}$ in diameter, loaded with the NIR-664-N-succinimidyl ester fluorophore were prepared as previously described. ${ }^{17}$

\section{Anti-C-reactive protein (CRP)/green fluorescent protein (GFP) - Nanoparticle coupling}

${ }_{65}$ Conjugation - PAMAM dendrimers (-COOH surface groups), generations $1.5,2.5,3.5$ and 4.5 were first activated with 1-Ethyl3-[3-dimethylaminopropyl]carbodiimide Hydrochloride (EDC) / $\mathrm{N}$-hydroxysulfosuccinimide (sulfo-NHS) mixture in $0.1 \mathrm{M} 2-(\mathrm{N}-$ morpholino)ethanesulfonic acid (MES) buffer, $\mathrm{pH} 4.5$ before 70 reacting with the protein. As an example, PAMAM G1.5 $(1 \mu \mathrm{mol}$, $16 \mathrm{x}-\mathrm{COOH})$ was dissolved in $0.5 \mathrm{ml}$ of MES buffer. To this solution, sulfo-NHS ( $24 \mu \mathrm{mol}, 1.5$ equiv. per one $-\mathrm{COOH}$ group) and EDC (96 $\mu \mathrm{mol}, 6.0$ equiv. per one $-\mathrm{COOH}$ group) were added; the final volume adjusted to $1 \mathrm{ml}$ with MES buffer and the 75 reaction was allowed to proceed for 15 minutes at room temperature. The NHS-activated dendrimer was then directly added into the NPs $(2 \mathrm{mg} / \mathrm{ml})$, while keeping the total volume at 1 $\mathrm{ml}$. This mixture was allowed to react for 30 minutes; subsequently, the excess of solution containing unreacted, free 80 dendrimer was removed by centrifugation $(15000 \mathrm{rpm}, 5$ minutes). The dendrimer-modified NPs were re-suspended in MES buffer, $\mathrm{pH} 4.5$ and the protein, either recombinant singlechain fragment anti-CRP antibody $(26 \mathrm{kDa}){ }^{19}$ or green fluorescent protein $(135 \mu \mathrm{g})$ was added. The reaction mixture was gently ${ }_{85}$ shaken for 4 hours at r.t., $100 \mu 1$ of MES buffer, $\mathrm{pH} 12.0$, added to hydrolyze the remaining NHS esters and the mixture was purified by centrifugation ( $4 \mathrm{x}, 15000 \mathrm{rpm}, 5$ minutes). The NPprotein bioconjugate was re-suspended in $0.1 \mathrm{M}$ PBS buffer, $\mathrm{pH}$ 7.4, with $0.01 \%(\mathrm{w} / \mathrm{v}) \quad \mathrm{NaN}_{3}$ and $0.5 \%(\mathrm{w} / \mathrm{v})$ of bovine serum 90 albumin (BSA). All reactions involving NPs were performed 
under reduced light conditions (reaction vessels wrapped up in aluminum foil) to prevent photo-bleaching and all final samples were stored in the dark at $4{ }^{\circ} \mathrm{C}$ before they were used in immunoassays.

${ }_{5}$ Longer term storage - For NP samples that are to be stored for longer than 30 hours before they are used in immunoassays or for shipping purposes, we highly recommend to aliquot the final solution into smaller $100-200 \mu \mathrm{L}$ fractions and freeze dry them under reduced light. The shelf-life of the antibody-sensitized NPs

10 can be thus significantly extended as supported by data shown in supporting information (ESI 1).

\section{Fluorescence measurements}

Direct binding assay - A 75 well plate was oxidized in oxygen plasma. The oxidation took place during $6 \mathrm{~min}$ in a plasma 15 chamber (400 Plasma System) at a working pressure of 0.26 mbar, $1000 \mathrm{~W}$ and with a flow of oxygen at $100 \mathrm{ml} / \mathrm{min}$. To adsorb antigen onto the surface for the particle-binding measurement, $5 \mu \mathrm{l}$ of the penta-valent antigen, CRP $(50 \mu \mathrm{g} / \mathrm{ml})$ solution, was loaded into each well and incubated at $37{ }^{\circ} \mathrm{C}$ for 20 two hours. The surface was then washed $1 \mathrm{x}$ with PBS containing Tween $20(0.2 \%, v / v)$ and further $1 \mathrm{x}$ with PBS. The plate was subsequently immersed in a PBS solution containing BSA (1\%, $\mathrm{w} / \mathrm{v}$ ) for one hour. For the assay kinetics determination, after rinsing the wells with PBS (1x) and drying under a stream of $\mathrm{N}_{2}$, 25 anti-CRP - NP biocojugates were added $(5 \mu \mathrm{l}$ of a $0.1 \mathrm{mg} / \mathrm{ml}$ suspension: $0.25 \mathrm{nM}$ assuming a density of $2.4 \mathrm{~g} . \mathrm{cm}^{-3}{ }^{20}$ and diameter of $80 \mathrm{~nm}$ ) and incubated at $37^{\circ} \mathrm{C}$ for various time. The plate was subsequently washed with PBS Tween $(0.2 \% \mathrm{v} / \mathrm{v})$ solution $2 \mathrm{x}$, with PBS once and dried under a stream of $\mathrm{N}_{2}$. ${ }_{30}$ Fluorescence measurements were performed on a Safire (Tecan) microplate reader. For NIR-doped NPs, the excitation and emission wavelengths were set at 672 and $700 \mathrm{~nm}$, respectively. The data were fitted using a power function according to equation 6. The non-specific binding was determined by extrapolation 35 from the fitted curve at the time $t=0$.

NP surface activity assessment - GFP was conjugated to the NP surface using PAMAM dendrimers, generations $1.5-4.5$ as described in the 'Anti-CRP/GFP - Nanoparticle coupling' section. The average surface coverage was measured by means of 40 fluorescence. The emission of GFP (508 nm) was clearly distinguishable from that of the NPs, which have emission maximum at $702 \mathrm{~nm}$. A calibration curve of GFP fluorescence at 8 different concentrations was constructed. The amount of bound GFP was calculated based on the known fluorescence yield per 45 molecule ${ }^{21,22}$ and converted to an area using the known dimensions of GFP. These figures then allowed the fractional surface coverage to be calculated using the known dimensions of the dendrimer.

Measurement of equilibrium constant for antibody binding to ${ }_{50}$ the antigen-sensitised reaction well - The equilibrium constant was estimated by measuring the fluorescence signal at equilibrium, resulting from incubation of the CRP-sensitised surface with lissamine rhodamine-labelled anti-CRP ScFv. Briefly, a 75 well plate was oxidized in oxygen plasma as ${ }_{55}$ described above. To load antigen onto the surface for the particlebinding measurement, $3 \mu 1$ of the penta-valent antigen, CRP (50 $\mu \mathrm{g} / \mathrm{ml}$ ) solution, was loaded into each well and incubated at $37^{\circ} \mathrm{C}$ for two hours. The surface was then washed $1 \times 10$ min with PBS containing Tween $20(0.2 \%, \mathrm{v} / \mathrm{v})$ and further $1 \times 5$ min with ${ }_{60}$ PBS. The plate was subsequently immersed in a PBS solution containing BSA $(1 \%, w / v)$ for one hour. After rinsing with PBS $(1 \times 10 \mathrm{~min})$, water $(1 \times 5 \mathrm{~min})$ and drying under a stream of $\mathrm{N}_{2}$, lissamine rhodamine-labelled anti-CRP was added at concentrations ranging from $0.1 \mathrm{ng} / \mathrm{mL}$ to $100000 \mathrm{ng} / \mathrm{mL}$, in 10 ${ }_{65}$ fold serial dilutions. The 75 well plate was then incubated at $37^{\circ}$ $\mathrm{C}$ for 1 hour. The plate was subsequently washed with PBS Tween $(0.2 \% \mathrm{v} / \mathrm{v})$ solution $2 \times 10 \mathrm{~min}$, with PBS $1 \times 5 \mathrm{~min}$ and dried under a stream of $\mathrm{N}_{2}$. Fluorescence measurements were performed on a Safire (Tecan) microplate reader. The signal fitted 70 to a Langmuir isotherm, with surface binding equilibrium constant $(9.5 \pm 2) \times 10^{5} \mathrm{M}^{-1}$ (as shown in Online Resource 1). The measurement highlighted significant variability of the capture plate surface preparation.

\section{Dynamic Light Scattering (DLS) measurements}

75 All NP dispersions were analysed at a nanoparticle concentration of $1 \mathrm{mg} / \mathrm{ml}$ in water. The stability of the dispersions was monitored at $25{ }^{\circ} \mathrm{C}$ over a period of 3 hours with the average of 50 measurements taken at two minute intervals. For the antigen mediated NP aggregation studies, $1 \mu 1$ of CRP solution (at a 80 concentration of $2.42 \mathrm{mg} / \mathrm{ml}$ in PBS) was introduced to the nanoparticle dispersion ( $1 \mathrm{ml})$. The DLS measurement was immediately started without further perturbation of the dispersion. All measurements were performed in duplicate with two different batches of sensitized NPs.

\section{${ }_{85}$ Theory of nanoparticle capture onto a surface by an antibody-antigen reaction}

A surface capture immunoassay can be considered in two ways: either incubation of antibody-sensitised particles with antigen followed by capture onto an antibody-sensitised surface of those 90 particles that have bound antigen; or capture of antigen onto an antibody-sensitised surface followed by capture of antibodysensitised particles onto the surface-bound antigen. In either case, the kinetics of reaction of a sensitised particle with a sensitised surface is a key element of the description of the ${ }_{95}$ process and hence of the assay design ${ }^{23,24}$. The size of the particles and the fraction of the particle surface that is active for the antibody-antigen reaction are the important parameters. In the present work, we have studied the capture of particles onto an antigen-loaded surface, thus focussing on the second stage of the 100 process, and for simplicity we have studied just one coverage of antigen on the capture surface since our aim has been to elucidate the effect of the linker on the activity of the particles in the assay. We have developed a simple kinetic model for particle capture onto an antigen-loaded assay surface by means of an antibody105 antigen reaction. This is similar to kinetic formulations treating single molecules ${ }^{25,26}$, but also accounts for the fact that each particle has a multiplicity of antibodies present on its surface. The NPs-surface-bound antibodies can be considered as active binding sites. Depending on the coverage of such active sites on 10 the particle, a collision of the particle with an antigen may or may not lead to reaction, with a probability proportional to the surface coverage of active sites on the particle and the surface coverage of antigen in the reaction well.

The capture reaction, of antibody-functionalised NPs onto the 
assay surface, can be written as:

$\mathrm{P}+\mathrm{S} \leftrightarrows \mathrm{PS}$

with forward rate constant $k_{\text {on }}$ and reverse rate constant $k_{\text {off }}$. Here, $\mathrm{S}$ denotes an active antigen site on the assay capture surface. If 5 the NP is bound to the surface by a single antibody-antigen interaction, then $k_{\text {off }}$ will simply be the dissociation rate constant for the antigen-antibody complex. The association rate, $k_{o n}$, will be determined by the probability of a reactive collision between an antibody functionalised NP and an unoccupied reactive 10 antigen site on the assay surface. A reactive collision, leading to coupling of the NP to the assay surface, would occur when a reactive part of the NP surface (an antibody, oriented with the binding site exposed) collides with a reactive part of the assay surface (an antigen, oriented with the epitope exposed). NPs are

15 in a continuous state of collision with the assay surface, at a rate determined by the diffusion coefficient of the NPs (hence by their radius and the viscosity of the reaction medium) and by their concentration. In the absence of any forces favouring a particular orientation of the NP with respect to the assay surface during

20 approach and collision, the probability that the collision will be with an active site on the NP surface will simply be the fraction of the NP surface area that is covered by active antibody. By 'active antibody' is meant an antibody molecule oriented on the NP surface such that its binding site is exposed and available for 25 reaction. The active antibody is only some fraction of the total antibody present on the NP surface, the fraction varying primarily with the surface coverage of antibody on the NP and dependent also on the means of attachment to the NP surface. The active fraction could decrease drastically as total antibody loading on 30 the surface increases ${ }^{26,27}$. As the NP concentration on the assay surface builds up, particles not only occupy sites but also physically block the assay surface area, on account of their size. The probability of obtaining a successful reactive collision between particle and capture surface also depends on the fraction 35 of the capture surface that is active for reaction. Therefore, the probability that the collision will be with an active site on the assay capture surface will be a product of the following:

- The fraction of the total assay capture surface that is covered by accessible antigen with the binding epitope correctly exposed to facilitate antibody binding;

- The fraction of the total assay surface that is unblocked by bound NPs;

- A coverage-dependent factor that expresses the requirement that a NP requires a space whose smallest dimension is at least as large as the NP diameter, in order that the NP can fit into the space.

This statement assumes that the approach and collision of nanoparticles is unaffected by the presence of previously bound NPs except insofar as these occupy surface area. If $k_{\text {off }}$ is ${ }_{50}$ sufficiently low, then the requirement that NPs can fit into the spaces available leads to a limiting coverage - the 'jamming limit' - which is approximately $55 \%$ of the total surface area for random sequential irreversible adsorption of spherical objects. ${ }^{27}$ If the coverage of the assay capture surface by bound NPs is ${ }_{55}$ sufficiently low, then the effects of particles jamming the surface, and of other particle-particle interactions, can be ignored. and the increase of NP coverage, and hence of surface-bound fluorescence will follow: $\frac{d \theta}{d t}=k_{o n} c(1-\theta)-k_{o f f} \theta 60$

where $c$ denotes the nanoparticle concentration in the solution at the capture surface and $\theta$ denotes the fraction of the total capture plate area that has been covered by particles: reaction can only ${ }_{65}$ occur on the un-covered area. The fraction of the total capture plate area that is obscured by a each particle depends on the size of the particle. Since the surface-bound fluorescence intensity, in the absence of self-quenching, will be proportional to the particle coverage $\theta: \quad I=F \theta$, then the fluorescence intensity variation $I$ 70 will also follow:

$$
\frac{d I}{d t}=F k_{o n} c-I\left(k_{o n} c+k_{o f f}\right)
$$

If the surface capture reaction does not lead to a significant 75 diminution of the particle concentration in the solution, so that $c$ can be considered constant, then the first-order rate equation (4) results:

$$
I=\left(\frac{F k_{o n} c}{k_{o n} c+k_{\text {off }}}\right)\left\{1-\exp \left(-\left(k_{o n} c+k_{\text {off }}\right) t\right)\right\}_{80}
$$

As coverage increases towards the jamming limit, then the rate of adsorption slows, shows an approximate power-law dependence on the surface coverage ${ }^{28}$ and also becomes dependent on the size $^{29}$, charge $\mathrm{e}^{30,}{ }^{31}$ and interaction potential ${ }^{32}$ of the captured ${ }_{85}$ particles. The effect of a slow desorption also would be to facilitate a slow rearrangement of the surface such that the coverage could further increase beyond the jamming limit. Also, if the capture rate were sufficiently great, then the concentration, $c$, of particles in the solution at the surface would decrease below 90 that in the bulk solution, far away from the surface, and a complete description should therefore include the effect of NPs diffusion towards the capture surface. Although it is a relatively simple matter to extend the treatment to include these effects, in this paper we consider only the reaction-controlled limiting 95 behaviour at low coverage, described by equation 4 .

The considerations of reaction probability that were discussed above are embedded in the capture rate constant, $k_{o n}$, which depends on both the fraction of the surface of the antigen-loaded capture plate that is in fact active for the capture reaction (that is, 100 it depends on the surface state of the adsorbed antigen) and on the fraction of the surface of the antibody-sensitised NP that is active for reaction. It can be written

$$
k_{\text {on }}=k_{D} \varepsilon \theta_{S} \theta_{P}
$$

${ }_{105}$ where $k_{D}$ is the diffusion-limit rate constant for NP consumption by the antigen-surface, dependent on the NP radius and the viscosity of the medium, $\varepsilon$ is the reaction efficiency for a reactive collision, $\theta_{S}$ is the fraction of the capture surface area (here the antigen-loaded capture plate) that is active for the capture 110 reaction, which in this case is dependent on the surface coverage of active adsorbed antigen on the prepared capture surface, and $\theta_{P}$ is the active fraction of the NP surface, in this case dependent on the surface coverage of active antibody attached to the NP. The central objective of surface functionalisation with dendrimer is to 115 increase $\theta_{P}$. We use eq 4 to deduce the dependence of the surface capture rate constant and hence $\theta_{P}$ on the generation number of 

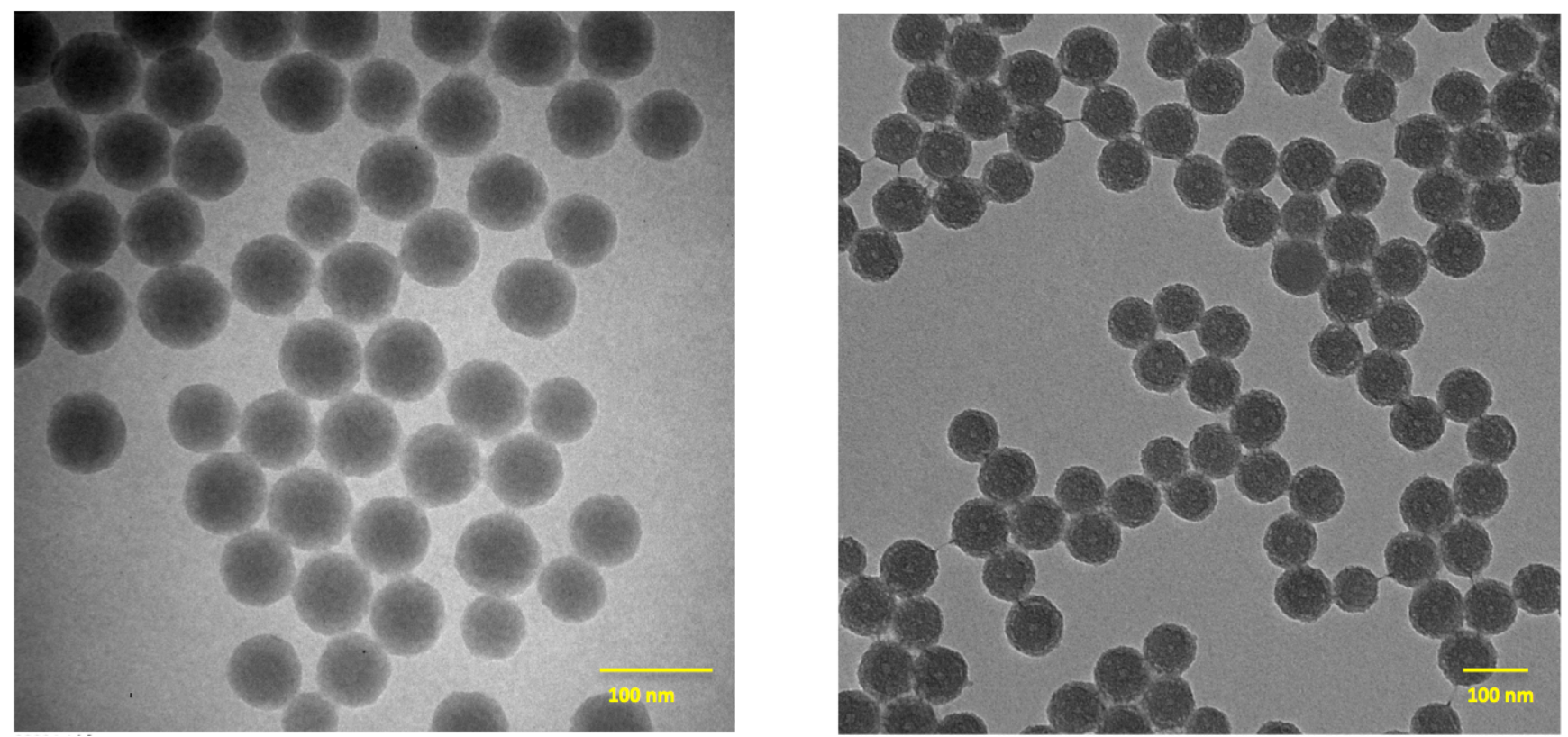

Fig. 2 TEM images of silica NPs, doped at $3 \% \mathrm{w} / \mathrm{v}$ of NIR-664 dye, taken few days after the sample was made (left image) and one year after storing the sample in $0.01 \mathrm{M}$ PBS buffer, $\mathrm{pH}=7.4$ (right image).

${ }_{5}$ dendrimer used for surface functionalisation. We do not in this communication address the question of maximising $\theta_{S}$ though clearly the same techniques that we use to increase the surface activity of the particles could also be used to increase the surface activity of the plate.

\section{${ }_{10}$ Results and Discussion}

A successful conjugation reaction between the particle and antibodies requires good monodispersity and colloidal/chemical stability of the particles, often in buffered solutions with relatively high salt concentration. The NPs used in this work were 15 prepared by reverse microemulsion method based on a water-inoil reverse microemulsion system. The optimal research scale of this method typically yields $20-30 \mathrm{mg}$ of NPs per batch and we have previously reported on batch-to-batch uniformity and polydispersion fidelity ${ }^{33}$ (for additional information on batch-to20 batch variations related to particle surface modification, see ESI 1). However, we also decided to elaborate on a longer term colloidal and chemical stability of the particles upon storage in aqueous solution. As demonstrated on figure 2, the reverse microemulsion method produces particles with low polydispersity 25 index (left TEM image). Interestingly, after one year in $0.01 \mathrm{M}$ PBS buffer, the particles retained reasonably good monodispersity (right TEM image). The particles showed a small degree of degradation and cross-bridging phenomenon, presumably due to hydrolysis and consequent polymerization of 30 surface bound silanes. The bridges between the particles were easily broken by ultrasonication. In order to avoid the aforementioned degradation and potential loss of fluorescence intensity, we recommend freeze-drying the samples as mentioned in Materials and Methods. Freeze-drying proved to be an 35 effective method for long term storage of even antibody-coated NPs (supporting information, ESI 2)

Another issue when preparing antibody-sensitized NPs is the purification of the reaction mixture. One of the simplest techniques to separate the antibody-coated NPs from the excess 40 of unrected antibody is by a sequence of centrifugationultrasonication steps. This method can introduce some degree of thermal stresses on the antibodies and may eventually compromise their activity. The desired level of purity is specific to an individual application. Complete removal of the excess 45 antibody from the reaction mixture can be achieved after four washing steps. However, about $99 \%$ of the unreacted antibody is already removed after two centrifugations-ultrasonications (supporting information, ESI 3)

According to the theoretical model presented in this 50 communication, the central objective is to increase the active surface area of the nanoparticles. Therefore, we checked that the average number of active carboxylate functions available for reaction after dendrimer sensitisation of the NPs scaled as expected with the dendrimer generation number. Green ${ }_{55}$ Fluorescent Protein (GFP) was conjugated to the NP surface using PAMAM dendrimers, generations $1.5-4.5$ and its surface coverage was then measured by means of fluorescence. The emission of GFP (508 nm) was clearly distinguishable from that 


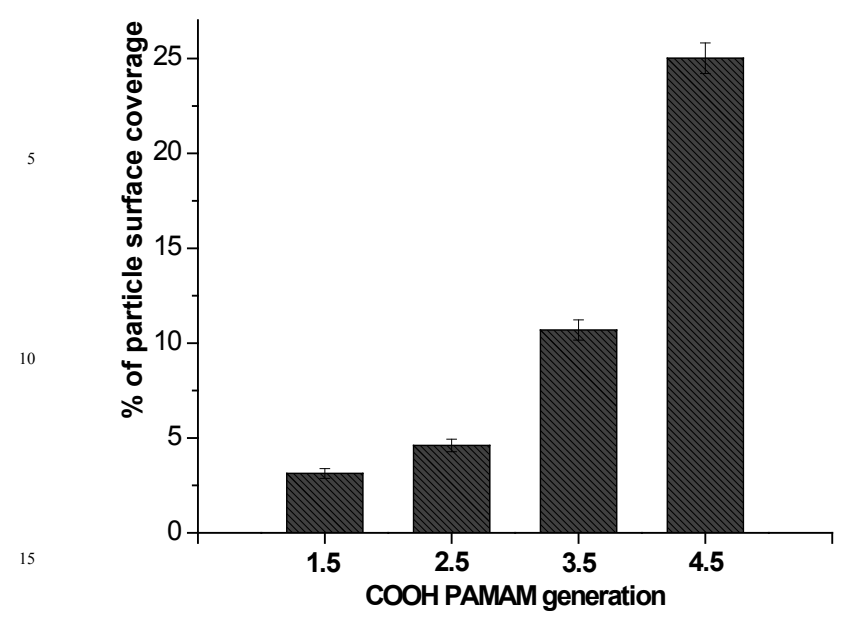

Fig. 3 Percentage of NP surface area covered with GFP after conjugation with four generations (1.5-4.5) of PAMAM dendrimers.

20

of the NPs. The amount of bound GFP was computed using the known fluorescence yield per molecule ${ }^{22,23}$ and converted to an area using the known dimensions of GFP. These figures then allowed the fractional surface coverage to be calculated using the 25 known diameter of the particles, augmented by the known dimensions of the dendrimer. Figure 3 illustrates the effect of dendrimer generation number on the amount of bound GFP protein. As expected, the amount of GFP conjugated to the NP surface increased with increasing generation number of the 30 dendrimer employed as linker, scaling approximately as the number of carboxylate groups in the outer shell of the dendrimer: the conjugation with PAMAM 4.5 (containing 128 surface carboxylate groups) resulted in a nanoparticle-GFP complex with at least an 8-fold higher protein loading than the same reaction 35 with the smallest dendrimer used, PAMAM 1.5 (containing 16 surface carboxylate groups).

In order to evaluate the effect of dendrimer generation number as a linker of antibodies to NPs, a direct binding fluorescence-linked immunosorbant assay (FLISA) was carried out. The antigen, ${ }_{40} \mathrm{CRP}$, was adsorbed onto the surface of microtitre plate wells.

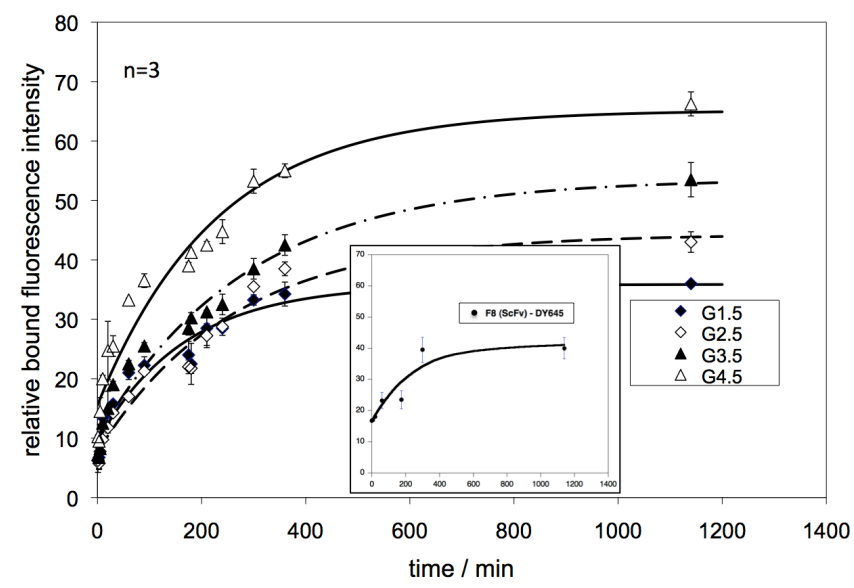

Fig. 4 Direct binding CRP assay curves for the nanoparticles sensitised with anti-CRP through PAMAM dendrimers.
45 The $\mathrm{ScFv}$ antibody was coupled to the fluorescent NPs and the effect of dendrimer linker generation number on the kinetics of capture of NPs onto the surface was measured.

Figure 4 shows the time variation of the relative fluorescence intensity from bound NPs. The only variable was the generation 50 number of the linking dendrimer: the capture surface preparation and the NP concentration remained the same for all measurements.

Table 1 Fitting parameters derived from curves in Fig. 4; and calculated $55 \mathrm{k}_{\text {on }}$ rate constants for antibody coupled directly to DY645 dye and antibody-sensitized NPs with four generations of PAMAM dendrimer

\begin{tabular}{ccccc}
\hline label & $\boldsymbol{I}_{\text {lim }}$ & $\boldsymbol{I}_{\text {offset }}$ & $\mathbf{1 0}^{5} \boldsymbol{k}_{\text {obs }} / \boldsymbol{s}^{-1}$ & $\boldsymbol{k}_{\text {on }} / \boldsymbol{M}^{-1} \boldsymbol{s}^{-1}$ \\
\hline DY645 & $24.9 \pm 3.1$ & $16.3 \pm 1.3$ & $7.1 \pm 1.9$ & $70 \pm 20$ \\
\hline NP-G1.5 & $26.4 \pm 1.9$ & $9.5 \pm 1.0$ & $10.0 \pm 1.8$ & $190 \pm 60$ \\
\hline NP-G2.5 & $36.6 \pm 2.1$ & $7.7 \pm 0.8$ & $6.5 \pm 0.75$ & $210 \pm 60$ \\
\hline NP-G3.5 & $43.5 \pm 2.4$ & $10.1 \pm 0.9$ & $5.9 \pm 0.7$ & $220 \pm 70$ \\
\hline NP-G4.5 & $49.6 \pm 3.2$ & $15.5 \pm 1.4$ & $7.3 \pm 1.0$ & $280 \pm 80$ \\
\hline
\end{tabular}

In our interpretation we have treated any non-specific adsorption of the particles to the surface as a time-independent offset of the ${ }_{60}$ fluorescence signal and therefore have modified equation 4 as follows, for fitting to the time variation of the fluorescence:

$I_{\text {rel }}=I_{\text {lim }, \text { rel }}\left\{1-\exp \left(-k_{\text {obs }} t\right)\right\}+I_{\text {offset }}$

${ }_{65}$ where $k_{\text {obs }}=k_{\text {on }} c+k_{\text {off. }}$. We take $I_{\text {offset }}$ to represent a non-specific adsorption of the antibody functionalised particles. Its variation across the different dendrimer generations used as linkers, compared with its value for a simple dye-linked antibody, is shown in Fig. 5B.

${ }_{70}$ One of our objectives was to compare the performance of the antibody-dendrimer linker conjugated NP with that obtained using antibody coupled directly to the fluorescent dye rather than to the dye loaded nanoparticles. Thus, in figures $5 \mathrm{~A}$ and $5 \mathrm{~B}$ we present the reaction parameters acquired by the non-linear least 75 square fitting of equation (6) to the experimental data for the dendrimer-antibody-conjugated NPs relative to those observed for an assay performed using dye-labeled antibody. The limiting value of relative fluorescence intensity at long time is:

$\left(I_{\text {lim }, \text { rel }}-I_{o f f s e t}\right)=F\left(\frac{k_{o n} c}{k_{o b s}}\right)^{80}$

The rate constant $k_{o b s}$ is obtained directly from the fit (eq 6). Thus:

${ }_{85} F\left(k_{\text {on }} c\right)=k_{\text {obs }}\left(I_{\text {lim,rel }}-I_{\text {offset }}\right)$

The scaling factor, $F$, was the same for all measurements as was the particle concentration, $c$. Therefore the values of $k_{\text {on }}$ scaled relative to one another can simply be obtained from the relative values of the product $k_{\text {obs }}\left(I_{\text {lim,rel }}-I_{\text {offset }}\right)$ and are shown in Fig. $5 \mathrm{~A}$. ${ }_{90}$ The relative 'on' rate, $\left(k_{o n}\right)_{\text {rel }}$ increased with increasing generation number of the dendrimer, consistent with the expected increase in the active surface area. The effect significantly out-weighed the 
effect of decreased diffusion coefficient of the nanoparticle relative to that of the dye-coupled antibody (through the parameter $k_{D}$ in equation 5). The effect was not, however, as great as that seen for the increase in total protein loading, consistent 5 with steric effects limiting access to the antibody binding sites ${ }^{28}$.

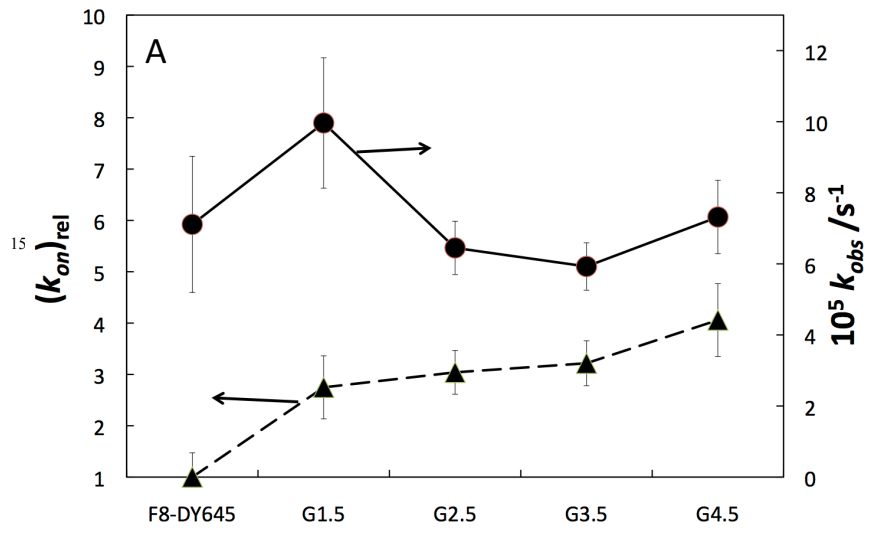

The fitted rate constant $k_{o b s}$ (Fig. 5A) varied only slightly with the generation number of the dendrimer and was, within the experimental uncertainty, the same for the dye-labelled and NPlabelled antibodies. It is justified below that $k_{\text {on }} c<<k_{\text {off }}$ so that 10 $k_{\text {obs }} \approx k_{\text {off. }}$. The lack of variation with dendrimer generation number

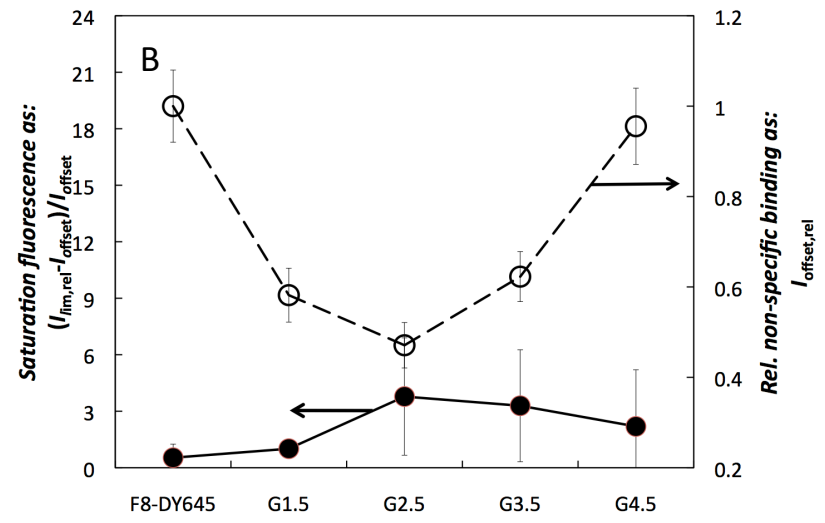

Fig. 5 (A) Rate constants for the surface-capture reaction. $\quad \mathbf{\Delta}$ : binding rate $k_{\text {on }}$ relative to the value for the antibody coupled directly to the fluorescent dye (F8-DY645); : fitted reaction rate constant, $k_{o b s} \approx k_{o f f} ;(\mathrm{B})-$ signal/background for the surface capture reaction. $\bigcirc:$ Fluorescence due to non-specific binding, relative to that found for the antibody coupled directly to the fluorescent dye (F8-DY645), $I_{o f f s e t, r e l}$; 0 saturation fluorescence relative to the non-specific binding, $\left(I_{\text {lim,rel }}-I_{\text {offset }}\right) / I_{\text {offset }}$

25 is as expected from the introductory theoretical discussion. The observed 'off' rate is consistent with literature values for pentameric CRP - antibody interactions $\left(10^{-4}-10^{-5} \mathrm{~s}^{-1}\right)^{23-25}$. The surface binding equilibrium constant, $K_{L}$, obtained from fitting of a Langmuir isotherm to the equilibrium adsorption of the 30 molecular dye-labelled antibody, gives the ratio $K_{L}=k_{\text {on }} / k_{\text {off }}$ for this antibody. Hence, using the mean estimate for $k_{\text {off }}=(7.4 \pm$ 1.6) $\times 10^{-5} \mathrm{~s}^{-1}$ gives for this antibody $k_{\text {on }}=70 \pm 20 \mathrm{M}^{-1} \mathrm{~s}^{-1}$. Literature values for $\mathrm{ScFv}$ antibodies interacting with pentameric CRP are typically $k_{\text {on }} \sim 2 \times 10^{4} \mathrm{M}^{-1} \mathrm{~s}^{-124}$. The discrepancy can be 35 resolved through consideration of equation 5. The key factor is the fraction of the antigen loaded onto the capture plate surface that is active for reaction: $\theta_{S}$. In the literature, the capture surface was prepared by adsorbing streptavidin first, followed by biotinylated antibody. It is not surprising that the value of $\theta_{S}$ 40 achieved by this procedure should be the factor of $10^{2}$ greater than that obtained as a consequence of the simple adsorption protocol that we have employed, that is implied by the relative values of $k_{o n}$. The 'on' rate constants for the sensitised particles can be calculated, given the relative rate constants deduced from 45 the capture kinetics. The values are given in Table 1 . The estimated errors accumulate through the calculations so are significantly larger than those for the relative rate constants. The deduced values for the product $k_{o n} c$ are at most $10^{-7} \mathrm{~s}^{-1}$, justifying the assumption $k_{\text {on }} c<<k_{\text {off }}$ used to identify $k_{o b s}$ with $k_{\text {off }}$.

${ }_{50}$ The other important characteristic of the labels to consider for use in an assay is the ratio of signal to background: in this case, the ratio of the equilibrium fluorescence (offset corrected) to the nonspecific offset (Fig. 5B). Again, a significant advantage is noted for the NP-labelled antibodies. The offset was not affected and, ${ }_{55}$ indeed, may have been reduced. The signal was significantly enhanced so the relative value of signal/non-specific background was increased by a factor of $3-7$ as a consequence of the use of the dendrimer-sensitised NPs as the label.
The reactivity of the NP-labelled antibodies can also be ${ }_{60}$ determined by measurement of the aggregation induced by the addition of the penta-valent CRP antigen to the antibodyactivated particle dispersion. This determination has the advantage of studying the particle chemistry alone, independent of any capture plate sensitisation protocol. We used a dynamic ${ }_{65}$ light scattering (DLS) measurement ${ }^{34,35}$ to determine average cluster volume as a function of time following the introduction of the antigen as depicted on Fig. $1^{36}$, and hence compare the reactivity of NPs activated with the different dendrimer linkers. The measurement also gave some further information concerning 70 the antibody-antigen reaction, in particular the parameter $\varepsilon$, the reaction efficiency, in equation 5 .

In general, there are two types of aggregation. ${ }^{37,38}$

- Diffusion-limited aggregation - aggregation where there is no barrier, interpreted as $\varepsilon=1$ : the average cluster size (mass or volume) increases linearly with time. Cluster mass distribution is flat up to a characteristic value then decreases exponentially.

- Reaction limited aggregation - aggregation where there is some barrier, interpreted as $\varepsilon<1$ : the rate is determined by a rate constant to surmount the barrier. The rate is also dependent on the surface concentration of reactive sites, which grows rapidly as the cluster expands. Average cluster size increases exponentially with time. Cluster mass distribution proceeds as $M / M_{\text {tot }}$ $\sim M^{3 / 2}$ and its theoretical behaviour is illustrated as a dashed line in Fig. 6, with an indication of the expected slope. $^{38,39}$

Figure 6 and 7 illustrate that the G3.5- and G4.5-functionalised particles showed the general characteristics of reaction-limited 90 aggregation in the presence of CRP. The literature values for association rate constants $\left(\sim 10^{4} \mathrm{M}^{-1} \mathrm{~s}^{-1}\right)^{24}$ are relatively small and would indeed be consistent with the presence of a barrier to 


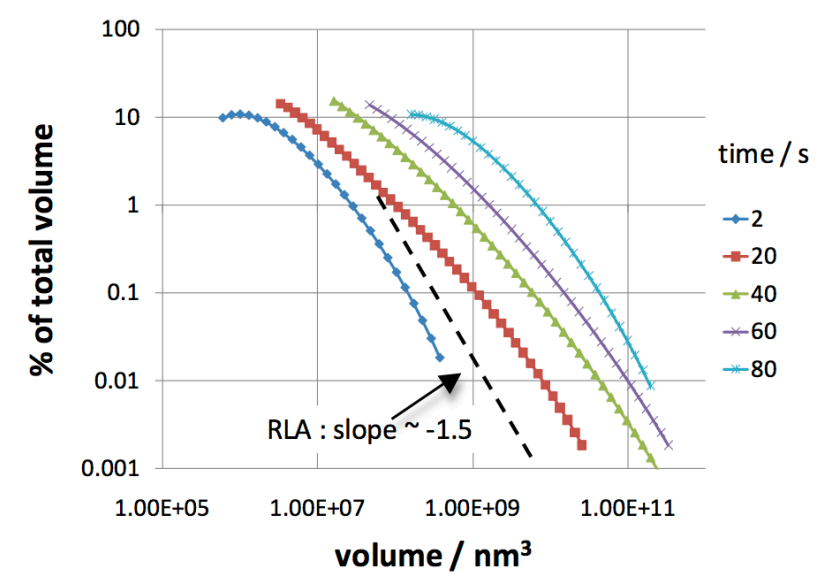

Fig. 6 Evolution of particle volume distribution with time in the CRPinduced aggregation of anti-CRP sensitised dendrimer G4.5-linked nanoparticles

reaction. The aggregation rate constant for the G4.5 particles was 5 some 5.6 times greater than for the G3.5 particles. The exponential increase of average cluster volume with time, in the reaction-limited aggregation of antibody-conjugated NPs in the presence of CRP antigen, can be described in terms of the probability of capture of antigen onto the surface of a NP or onto 10 a NP at the outside of a cluster, times the probability that this site will collide with an active site on another NP or cluster. Thus, the rate constant for average cluster volume should increase approximately as $\left(\theta_{P}\right)^{2}$ for a given initial concentration of NPs and antigen. Therefore, the aggregation rate enhancement for the ${ }_{15}$ G4.5 NPs compared with the G3.5 NPs implies $\theta_{P}$ for the G4.5 NPs to be about twice that of the G3.5 NPs. This effect is indeed consistent with the increase in protein loading indicated in figure 3 , but is rather greater than that observed for the surface capture FLISA, for which the capture rate enhancement (Fig. 5A) was 20 about $30 \%$. A plausible explanation is that the steric constraints for capture of a NP by the antigen adsorbed onto the microtitre plate were significantly greater than for the antigen-mediated NP aggregation in solution.

\section{Conclusions}

${ }_{25}$ Overall, the potential of dye-doped NPs as new generation labels can be realized only if the biomolecule in question is immobilised at the appropriate surface density while maintaining its activity. Moreover, the measured signal should be maximised relative to noise and background contributions, which in antibody-based 30 assays, is dependant on the background response from the nonspecific absorption. In this communication, we have demonstrated how the use of PAMAM dendrimers as multivalent linkers effectively controls and maximises the active fraction of the particle surface. PAMAM dendrimers have been

35 demonstrated as efficient coupling agents to conjugate antibodies to the surface of dye-doped silica nanoparticles. An antigeninduced aggregation and a direct binding FLISA were used to demonstrate the effect of the growing number of terminal functional groups of the dendrimers on the resulting assay rate.

${ }_{40}$ The protein binding capacity of the dendrimer-sensitised NPs increased by the same factor as the number of surface carboxylate groups on the dendrimer used. The reaction rate in an antigen-

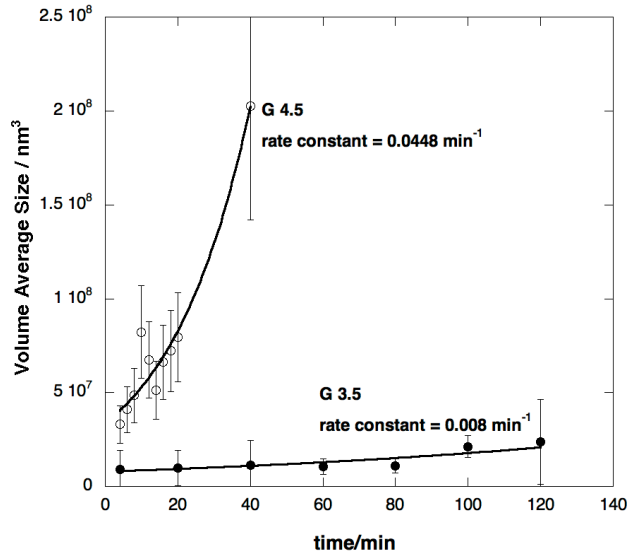

Fig. 7 Time evolution of the volume-average aggregate size time in the ${ }_{45}$ CRP-induced aggregation of anti-CRP sensitised dendrimer linked nanoparticles. G3.5 linker: $\bullet$; G4.5 linker: $\bigcirc$. The exponential increase with time is as expected for reaction-limited aggregation.

induced aggregation also increased by the same factor. The reaction rate and signal enhancement in a surface capture 50 immunoassay, resulting from the use of dendrimers as linkers, was significant but less than that found for antigen-induced aggregation, the effect being plausibly attributed to steric effects limiting the efficiency of the surface capture reaction. The highest generation of the PAMAM dendrimers (G4.5) used in this study 35 showed the highest surface binding rate and the highest signal in the direct binding FLISA. The effect of increased active antibody loading significantly outweighed any effect of a decreased NP diffusion coefficient compared to that of a molecular dye-labelled antibody. The practically important parameter, the ratio of the ${ }_{60}$ equilibrium fluorescence (offset corrected) to the non-specific offset, or signal to background ratio, increased by a factor of $\sim 4$ for the G4.5 dendrimer-conjugated NPs compared to the molecular dye-labelled antibody.

\section{Acknowledgements}

${ }_{65}$ This material is based upon research supported by the Science Foundation Ireland under Grant No. 05/CE3/B754. CL acknowledges the EU Seventh Framework Programme for support in the form of a Marie Curie Re-Integration Grant. DEW acknowledges the award of an E.T.S. Walton Visiting Fellowship 70 of Science Foundation Ireland. We thank Niamh Gilmartin and Meg Walsh for kindly providing GFP.

\section{Notes and references}

${ }^{a}$ Biomedical Diagnostics Institute (BDI) Dublin City University, Collins Avenue, Glasnevin, Dublin 9, Ireland. Fax: +3531700 6558; Tel: +353 751700 6337; E-mail: vladimir.gubala@dcu.ie

${ }^{b}$ School of Biotechnology, Dublin City University, Dublin 9, Ireland, Fax: +3531700 6558; Tel: +3531700 7810; E-mail:

richard.okennedy@dcu.ie, stephen.hearty@dcu.ie,

barry.mcdonnell3@mail.dcu.ie

${ }_{80}{ }^{c}$ MacDiarmid Institute for Advanced Materials and Nanotechnology, Department of Chemistry, University of Auckland, Private Bag 92019, Auckland 1142, New Zealand. Fax: +64 4463 5237; Tel: +64 9373

7599; E-mail: david.williams@auckland.ac.nz

$\uparrow$ Electronic Supplementary Information (ESI) available: [ESI 1 ${ }_{85}$ Nanoparticle synthesis - polydispersity index variations related to particle 
surface modifications, ESI 2 - Storage of protein-sensitized nanoparticles, ESI 3 - Washing the reaction mixture by ultrasonication, ESI 4 Measurement of equilibrium constant for antibody binding to the antigensensitised reaction well]. See DOI: 10.1039/b000000x/

1. B. McDonnell, S. Hearty, P. Leonard and R. O'Kennedy, Clin. Biochem. 2009, 42, 549-561.

2. P. M. Ridker, N. Rifai, N. R. Cook, G. Bradwin and J. E. Buring, Jama-J. Am. Med. Assoc., 2005, 294, 326-333.

10 3. Z. H. Li, R. B. Hayman and D. R. Walt, J. Am. Chem. Soc., 2008, 130, 12622.

4. M. L. R. Lim, M. G. Lum, T. M. Hansen, X. Roucou and P. Nagley, J. Biomed. Sci., 2002, 9, 488-506.

5. S. Nagl, M. I. J. Stich, M. Schaferling and O. S. Wolfbeis, Anal. Bioanal. Chem., 2009, 393, 1199-1207.

6. R. S. Davidson and M. M. Hilchenbach, Photochem. Photobiol., 1990 52, 431-438

7. C. S. Thaxton, D. G. Georganopoulou and C. A. Mirkin, Clin. Chim Acta, 2006, 363, 120-126.

20 8. A. Burns, H. Ow and U. Wiesner, Chem. Soc. Rev., 2006, 35, 1028 1042.

9. C. E. Fowler, B. Lebeau and S. Mann, Chem. Commun., 1998, 18251826.

10. C. McDonagh, O. Stranik, R. Nooney and B. D. MacCraith, 25 Nanomedicine, 2009, 4, 645-656.

11. J. L. Yan, M. C. Estevez, J. E. Smith, K. M. Wang, X. X. He, L. Wang and W. H. Tan, Nano Today, 2007, 2, 44-50.

12. R. P. Bagwe, L. R. Hilliard and W. H. Tan, Langmuir, 2006, 22, 4357 4362.

30 13. M. X. Liu, H. F. Li, G. Luo, Q. F. Liu and Y. M. Wang, Arch. Pharmacal Res., 2008, 31, 547-554.

14. L. O. Hansson, M. Flodin, T. Nilsen, K. Caldwell, K. Fromell, K. Sunde and A. Larsson, J. Immunoassay Immunochem., 2008, 29, 1-9.

15. J. S. Tan, D. E. Butterfield, C. L. Voycheck, K. D. Caldwell and J. T. Li,

35 Biomaterials, 1993, 14, 823-833.

16. A. Hucknall, S. Rangarajan and A. Chilkoti, Advanced Materials, 2009, 21, 2441-2446

17. V. Gubala, X. L. Guevel, R. Nooney, D. E. Williams and B. MacCraith, Talanta, 2010, 81, 1833-1839.

40 18. C. A. Labarrere and G. P. Zaloga, Am. J. Med., 2004, 117, 499-507.

19. P. Leonard, P. Safsten, S. Hearty, B. McDonnell, W. Finlay and R. O'Kennedy, J. Immunol. Methods, 2007, 323, 172-179.

20. R. K. Iler, The Chemistry of Silica: Solubility, Polymerization, Colloid and Surface Properties and Biochemistry of Silica, John Wiley and

45 Sons, Chichester, 1979.

21. M. Zimmer, Chem. Rev., 2002, 102, 759-781.

22. B. S. Melnik, T. V. Povarnitsyna and T. N. Melnik, Biochem. Biophys. Res. Commun., 2009, 390, 1167-1170.

23. R. V. Olkhov and A. M. Shaw, Anal. Biochem., 2010, 396, 30-35.

50 24. D. H. Choi, Y. Katakura, K. Ninomiya and S. Shioya, J. Biosci. Bioeng., 2008, 105, 261-272.

25. M. L. M. Vareiro, J. Liu, W. Knoll, K. Zak, D. Williams and A. T. A Jenkins, Anal. Chem., 2005, 77, 2426-2431.

26. H. Xu, J. R. Lu and D. E. Williams, J. Phys. Chem. B, 2006, 110, $1907-$ 1914.

27. E. L. Hinrichsen, J. Feder and T. Jossang, J. Stat. Phys., 1986, 44, $793-$ 827.
28. P. Schaaf and J. Talbot, Phys. Rev. Letters, 1989, 62, 175-178.

29. B. Senger, P. Schaaf, J. C. Voegel, A. Johner, A. Schmitt and J. Talbot, J. Chem. Phys., 1992, 97, 3813-3820.

30. M. R. Oberholzer, J. M. Stankovich, S. L. Carnie, D. Y. C. Chan and A. M. Lenhoff, J. Colloid Interface Sci., 1997, 194, 138-153.

31. Z. Adamczyk and P. Weronski, Adv. Colloid Interface Sci., 1999, 83, 137-226.

65 32. M. R. Oberholzer, N. J. Wagner and A. M. Lenhoff, J. Chem. Phys., 1997, 107, 9157-9167.

33. R. I. Nooney, C. M. N. McCahey, O. Stranik, X. L. Guevel, C. McDonagh and B. D. MacCraith, Anal. Bioanal. Chem., 2009, 393, 1143-1149.

34. H. Jans, X. Liu, L. Austin, G. Maes and Q. Huo, Anal. Chem., 2009, 81, 9425-9432.

35. M. Kavitha, M. R. Parida, E. Prasad, C. Vijayan and P. C. Deshmukh, Macromol. Chem. Phys., 2009, 210, 1310-1318.

36. G. K. Vonschulthess, G. B. Benedek and R. W. Deblois, Macromolecules, 1980, 13, 939-945.

75 37. M. Lattuada, H. Wu, P. Sandkuhler, J. Sefcik and M. Morbidelli, Chem. Eng. Sci., 2004, 59, 1783-1798.

38. M. Y. Lin, H. M. Lindsay, D. A. Weitz, R. Klein, R. C. Ball and P. Meakin, J. Phys. Cond. Matter, 1990, 2, 3093-3113.

39. R. C. Ball, D. A. Weitz, T. A. Witten and F. Leyvraz, Phys. Rev. Letters, $80 \quad 1987,58,274-277$. 